\title{
A Preliminary Study of the Translation of English Idiomatic / Formulaic Expressions by ESL/EFL Students: As Marked and Non-Canonical Forms
}

\author{
Ayman Nazzal \\ English Department, Faculty of Humanities \\ An-Najah University, Nablus, Palestine
}

\begin{abstract}
Recent studies done on the translation of "idiomatic/formulaic expressions" have always concerned themselves with the type of strategies used to account for the translation of idiomatic expressions. However, the difficulties which ESL/EFL students encounter in tackling such expressions stem not only from finding the appropriate strategy but also from the complex and culture-specific nature of such expressions which seem to constitute a real challenge to both native and non-native speakers
\end{abstract}

This paper is two-folds: First, it argues for the proposition that idiomatic /formulaic expressions can potentially represent the 'marked' rather than the 'unmarked' or the 'non-canonical versus canonical 'structures in English or any language and as a result of that such expressions constitute a real challenge to ESL/EFL students. It attributes the difficulties that such expressions pose to ESL/EFL students to the peculiar nature and recurrent structure of such expressions. Second, it provides a sound rationale for ESL/EFL students' adoption of specific translation strategy over other one to account for the translation of such expressions.

The methodology in this study consists of a set of 20 idiomatic expressions some of which have equivalence in the students' native language while other expressions do not have equivalents. The test is administered to 71 undergraduate English major at An-Najah National University. The second component of the test has dealt with the interview which the instructor conducted as a follow -up and for the sake of soliciting necessary feedback concerning students' responses and the opting of specific translation strategy over other possible ones.

The findings of this study have shown that ESL/EFL students have relied heavily on their mother tongue as a potential strategy to account for the translation of idiomatic/formulaic expressions. Their adoption of the literal translation strategy over other possible strategies is a clear indication that EFL/ESL students resort to their mother tongue to account for the translation of such expressions due to lack of pragmatic/cultural competence and lack of exposure to the use of such idioms. The findings have also underscored a) the merits and significant role which the Cross-Linguistic Influence known as CLI can play in any learning process; and b) the Markedness Deferential Theory known as MDT as an instrumental mechanism in assisting ESL/EFL students and instructors with the task of distinguishing between " marked and unmarked" structures.

Keywords: Inter-Lingual Transfer, Idioms, Cross-Linguistic Influence (CLI), Universal Grammar (UG), Marked-ness Deferential Theory (MDT), and Canonical versus Non-Canonical Structures.

\section{INTRODUCTION}

There is a great deal of wisdom which one can draw from the opening statement in Wray's book on Formulaic Language and the Lexicon concerning the peculiar nature of idiomatic/formulaic expressions which inclines one to believe that their acquisition is likely to be different from that of any typical linguistic forms which at first glance might surprise us but there is some truth in it if one ponders the several important aspects about their structure and the disparity between their conceptual and associative meanings which such expressions conjure up in the mind of native speakers. We are probably hard pressed to arrive at the conclusion that idiomatic/formulaic utterances are not only distinct from non-formulaic utterances but may also call for the development of a different type of competence and the adoption of a specific pedagogical approach for their acquisition. This inclines one to make the proposition that they are really different from typical or ordinary language structure. In fact, Wray has eloquently and explicitly described such expressions in the following statement: "The common link between formulaic languages across different speakers might even not be linguistic at all." (P. ix 2002) 
Wray's comments concerning the nature of such expressions seem to be consistent with the precise definition provided by Longman Dictionary (1998) which defines an idiomatic expression as " a sequence of words which has a different meaning as a group from the meaning it would have if you understand each word separately" (p.vii). Other linguists like Lewis who provides another definition which appears to fit the very nature and lexical structure of such expressions. He states that an idiom is "a multi-word lexical item where the meaning of the whole is not directly related to the meanings of the individual words" (1998: 217).

By and large, part of the difficulty which non-native speakers encounter in their translation of idiomatic/formulaic expressions stems from the assumption that such expressions are different from their linguistic counterparts on account that ordinary linguistic utterances can undergo various transformational processes; and by virtue of that ordinary linguistic utterances are not recurrent utterances but they are creatively produced utterances and subject to a set of rules and structures. And thus acknowledging the existence of a clear disparity between them lends support to the initial proposition that they are different and require students /learners to tap on different mental resources to be able to comprehend them and use them adequately when needed in social interaction (Wray 2002).

While there are many reasons for such a problem, an essential part of the difficulty is that ESL/EFL students seem most frequently to appeal to the conventional/conceptual/semantic meaning to account for the translation of such expressions upon hearing them from native or non-native speakers. To explicate this further an idiomatic expression like," eat your heart out", would be a real challenge for EFL/ESL students to pin down its intended/idiomatic meaning since appealing to the literal or conceptual (semantics) meaning would not assist students in deducing the idiomatic meaning of such an expression nor would the figuring out of the individual meaning of the words together which constitute the meaning of the whole expression do them any good.

Another difficulty that EFL/ESL college students face with the use of such expressions stems from their lack of pragmatic competence which manifested itself in students' responses to various idiomatic expressions. It is noted that even when they know the meaning of some of these expressions, they fail to use them properly in their social context on account that they have little exposure to the way native speakers use such expressions in daily social interaction (see, Pawley and Syder, 1983). According to Frank (2013) learning a foreign language involves the acquisition of adequate literacy in the target language and familiarity with its culture.

\section{LITERATURE REVIEW}

The question of formulaic/idiomatic expressions has been dealt with by various linguists but with varying levels of interests and enthusiasm. Some linguists have underscored the acquisition of such expressions and their overall importance in social interaction (see, Hymes, 1968) while other linguists have not even accounted for them as part of one's cognitive system and by virtue of that their role is being underplayed in generative syntactic theory (see, Chomsky, 1993). Of those who have underscored its role and value is Hymes (1968) who claims that " a vast portion of verbal behavior ...consists of recurrent patterns, linguistic routines ...[including] the full range of utterances that acquire conventional significance for an individual, group, or whole culture" (pp. 126-127). Another linguist who has underscored the positive role of such expressions is Charles Fillmore (1979) who remarked that "a large portion of a person's ability to get along in a language consists in the mastery of formulaic utterances" (p. 92).

Other linguists have expressed a different opinion on the question of formulaic/idiomatic expressions. For instance Sperber and Wilson, (1995) claim that "idiomatic expressions can be considered a species of " Formulaic expressions" since the former shares a set of characteristics which can be applied to formulaic expressions and by virtue of that they are usually rendered holistically in translation. Idiomatic expressions are highly important for ESL/EFL students to master since having an adequate level of literacy in them reflects mastery and high proficiency in a specific language (Wray, 20002).

Other linguists such as Sequeiros (2004) have expressed some serious concern about the way we deal with idiomatic/formulaic expressions on the basis that they vary from language to language and require specific type of competence to use them properly in translation. He has made the following invaluable comments concerning the nature of idiomatic/formulaic expressions: 
[formulaic] 'forms are, from a linguistic point of view, radically different from language to language and in most cases literal linguistic translation does not work. Normally there only is a limited set of forms for each type of expression, which may or may not be available (p.105)". He concluded that the translation of idiomatic expressions is extremely difficult and it is contingent upon students acquiring pragmatic competence.

Baker (1992: 63) claims that idiomatic expressions are, "frozen patterns of language which allow little or no variation in form, and in the case of idioms, often carry meanings which cannot be deduced from their individual components". She also remarked that any change in their original form/structure would render such expressions meaningless.

Awwad (1991) has pointed out the constraints which ESL/EFL student are destined to encounter upon translating them on account that they require the attainment of cultural and pragmatic competence by students/translators to use them effectively and correctly. In an effort to bridge the cultural difference between idioms, he provided a theoretical framework for the translation of idioms. However, such a framework which classifies idioms into four distinct categories, requires ESL/EFL students to develop their linguistic and pragmatic competence so that they can opt for the proper translation strategy to account for the various species of idioms. Such thing in and of itself constitutes a real challenge.

According to Pinker (1994) idiomatic expressions are ' prefabricated chunks of words' and so they are distinct from the 'unmarked forms'. However, the findings of this study point out that ESL/EFL students tackle the task of translating idiomatic expressions as if they are dealing with any form of language by appealing to semantics to account for their translation even though their acquisition appears to be distinct from that of the unmarked forms of language -an intriguing assumption which Obler's (1982) study has attempted to point out.

Recent studies on the translation of idiomatic /formulaic expressions have concerned themselves with finding the appropriate translation strategies on the basis that idiomatic expressions are so diverse and therefore they require different translation strategies to account for their translation (see, AbuSsuaydeh ,2004). Other studies have focused on providing a framework for students to account for the translation of such expressions (Awwad, 1991). While we acknowledge that finding the proper strategy is part of the existing problem, we think that the primary challenge that faces ESL/EFL students lies somewhere else. It has to do with the diversity of types of idioms and the complex nature of such expressions which has immensely contributed to such a challenge which this study attempts to point out and underscore. Other pertinent studies have also pointed out that deviation resulting from inter-lingual transfer is part and parcel of the EFL/ESL learning process (Gass and Selinker, 1983, 1994; Odlin, 1989).

There is no denying that the infrequent use of ESL/EFL students' and instructors' of such expressions in the learning/teaching process is a clear indication that such expressions constitute a real challenge to students by virtue of their very complex nature and as being distinct from ordinary linguistic forms which induce one to consider them as marked structures. Therefore enhancing their cultural competence is a key component for ESL/EFL students to become familiar with the target language. According to Frank (2013) learning a foreign language and acquiring a good literacy in it is prerequisite to becoming conversant in the target language.

The following part draws on Marked-ness Deferential Theory, Canonical Versus Non-canonical, and Cross-Cultural Linguistic Influence to account for the disparity between idiomatic versus nonidiomatic language. Such disparity appears to be the primary cause for the difficulty which students face to account for the translation of such expressions

\section{AsSumptions AND AnAlyses}

\subsection{Markedness \& Universal Grammar Theory}

This is another perspective which potentially has some bearing on the question of difficulty encountered by first and second language learner by appealing to the principles of universal Grammar. For instance, one of the most explicit ways to draw some assistance from Marked-ness Deferential Theory (referred to herein as MDT) is by affording learners the opportunity to distinguish between marked and unmarked categories in a specific language and by virtue of that language learners would minimize their efforts of acquiring language. 
For instance the application of MDT would afford students the opportunity to account for the distinction between regular (unmarked) forms and irregular (marked) forms and the merits which ESL/EFL students can learn from such an example by realizing the countless merits in terms of pointing out the source of difficulty that EFL college students encounter in the process of first and second language acquisition.

According to Eckman (1977) "M.D.T", can potentially account for the direction of difficulty by appealing to "universal Grammar (UG). Eckman points out that marked forms/structures are difficult to acquire in comparison with unmarked forms or structures on account that the unmarked forms/structures have wider distribution than the marked forms/structures and follow a set of specific rules and are mostly structure-dependent. For instance, one can easily argue that the acquisition or translation of idiomatic/formulaic expressions by ESL/EFL learner can potentially be an extraneous task (Wray 2002; Pinker 1999; Pawley \& Syder 1983), since one can consider the acquisition of such expressions to represent the marked forms of the target language on account that their distribution in any language is finite and bounded in comparison with non-idiomatic expressions.

Some linguists have focused on the utility and applicability of UG as an approach (Ekman, 1977; Comrie, 1990) or a framework of linguistic universals to account for first and second language acquisition. It has been claimed that such a possibility-the application of UG-can potentially constitute a mechanism with which linguists would be able to account for the parameters which distinguish native language from the target one.

I would not be making a hasty judgment to claim that MDT can potentially be instrumental in pointing out the potential constraints which EFL/ESL students can run into in their acquisition of any language. Take for instance the type of difficulty which EFL/ESL students face in dealing with the translation of idiomatic/formulaic expressions which may potentially lie in the disparity which exist between the conventional and non-conventional meaning of such expressions on the one hand and another potential constraint is whether such expressions have equivalents or not in the learner's first language.

There is some sort of analogy between the act of being able to account for the disparity between the conventional and non-conventional meaning of a specific expression and that of accounting for the distinction between unmarked versus marked forms in English. Suffice to say, appealing to MDT is highly instrumental and very sound since one can practically be convinced with its merits in predicting the potential problems which EFL/ESL learners are expected to encounter in the process of language acquisition and the direction of difficulty that they are destined to face.

Since MDT draws on some of the principles of Universal Grammar, then I assume that the merit of applying MDT to UG is that MDT can assume that certain parameters are language specific since they have less distribution and other parameters are language universal since they have large distribution in diverse languages and consequently they represent the unmarked category. Chomsky's view (1986) on this issue deserves to be cited:

"UG consists of various subsystems of principles. It has the model structure that we regularly discover in investigation of cognitive systems. Many of these principles are associated with parameters that must be fixed by experience. The parameters must have the property that they can be fixed by quite simple evidence, because this is what is available to the child; the value of head parameter, for example, can be determined from such sentences as John saw Bill (versus John Bill saw). Once the values of the parameters are set, the whole system is operative. The system is associated with a finite set of switches, each of which has a finite number of positions. Experience is required to set the switches. When they are set, the system functions." (p.146)

Let's take Wray's (2002) illustration as an example to explicate what is really meant by fixing one's parameters to be consistent with one's language. She cited the brand label for cereal Kellogg's 'rice crispy' and noted that when some native speakers of English were asked to think of what this label really meant, they were shocked to realize that it meant just rice. The reason why there was some confusion on the part of native speakers to account for the idiomatic meaning of such an expression has to do with the fact that had the brand name been written and advertized in the canonical form/structure as 'crispy rice', it would not have caused the confusion it had in my opinion. Of course it is intentionally written in a non-canonical and marked way 'rice crispy' to draw the attention of consumers and entice them to the product. 
Most commercial ads are drafted in a way to entice customers to try the product. For An English native speaker the canonical form of a noun phrase is a linguistic form/structure in which the modifier if exists, precedes the modified head noun. 'rice crispy' is the marked and non-canonical form/structure since it is being reversed and derived from its base structure: crispy rice. The confusion over its meaning has resulted from a deviation of a head-initial to head-final parameter which native speakers are not accustomed to.

Based on Chomsky's (1993) views on our cognitive system the question of idiomatic /formulaic expressions appears to have no place despite the creativity and unbounded aspects of our cognitive system. This implies that our cognitive system can be very intriguing to the point that it cannot be accounted for all the time. Chomsky (1993) seems to be saying that part of the knowledge that one possesses is inborn and that variation among people is inevitable and that is due to one's upbringing which appears to have a real impact on one's performance system. This inclines us to accept the proposed proposition that formulaic/idiomatic expressions are different from their linguistic counterpart and thus buttressing the initial proposition that they are different and require students /learners to tap on different mental resources to be able to acquire some literacy in them and use them when needed in social interaction

\subsection{Canonical Versus Non-Canonical Expressions}

Kaplan's dichotomy may apply eloquently to idiomatic/non-idiomatic expressions. According to Kaplan, (1995) sentences which deviate from the following syntactic structure: NP VP Formula would be considered non-canonical. The acronym NP stands for a noun phrase constituent while the acronym VP stands for a verb phrase constituent. For instance the sentences in set A are syntactically considered canonical whereas those in set B are considered non-canonical:

Set A: 1 . George is a student of linguistics

2. George wants Alice to stay.

Set B: 1 . George is a student of linguistic, isn't he?

2. George would like to attend the party.

One can always claim that formulaic/idiomatic expressions can potentially be structure-bound in the sense that if one changes the word order of the 'prefabricated structure' of a particular idiomatic/formulaic expression, the outcome of such an operation is a meaningless expression (Baker 1992). Take for instance the idiom by and large and try to reverse its original structure, or add or delete anything from it or replace one word with another, it will mean nothing other than the meaning of each single morpheme. Therefore, formulaic/idiomatic expressions do not qualify to be part of 'canonical sentences on the basis that their structure does not adhere to what constitutes canonical sentences.

According to Baker (1992: 63) idiomatic expressions are "frozen patterns of language which allow little or no variation in form, and in the case of idioms, often carry meanings which cannot be deduced from their individual components". Therefore, the criteria which can apply to the dichotomy between canonical/non-canonical may apply to idiomatic/non-idiomatic expressions. There is some resemblance between non-canonical and idiomatic expressions in various ways one of which is that a canonical form follows the normal word order and structure of English sentences whereas the latter deviates from such a structure. A 'Prefabricated sequence' is an expression used by Wray (2002) to refer to formulaic sequence which refers to a set of constituent parts which is accepted to mean something in a specific community.

According to Kaplan (1995) non-canonical sentences are considered unusual due their structure even though they are syntactically derived from canonical sentences via a set of transformational rules. Non-canonical sentences would be a component of the 'marked' category since their distribution in any language is finite and they may not fully be familiar to all speakers of a specific language. Therefore, acquiring such forms requires much more effort and the adoption of a specific pedagogical mechanism. So for example the non-canonical sentence number 2 is derived from the canonical counterparts in sentence 1, via a transformation rule called 'reposing' as the following example illustrates this process: 
1. I can't stand lawyers.

2. Lawyers, I can't stand. This sentence is derived from the canonical sentence number (1) as a result of the application of a transformational rule called "reposing".

Suffice it to say, idiomatic expressions are recurrent expressions and a lot of the characteristics which apply to non-canonical forms apply to them and by virtue if that their translation poses a real challenge to ESL/EFL students.

\subsection{Pragmatics Versus Semantics}

According to Thomas (1983) there are two types of pragmatic failure: 'pragma-linguistic' and 'sociopragma-linguistic' failure. To explicate such a fine distinction, let's take the expression "thank you" as an example cited in Sana's unpublished thesis (2009) which illustrates the fact that failure to recognize the pragmatic force of this phrase, is considered a pragmatic failure; however, not knowing when, how and for what reason is considered a socio-pragmatic failure. For example saying "thank you" in a specific situation in a particular culture implies that the speaker has received some assistance and that he/she is grateful for such thing. But the same utterance at the same context may be uttered and implies an offensive act particularly when the recipient does not need the giver's assistance, which amounts to a rejection of an offer. This is an illustration of pragmatic transfer because the speaker transfers L1 culture-bound peculiarities into L2 situation. This inevitably leads to communication breakdown. What is misunderstood here is the intended meaning of the speech act of thanking in specific situation.

Instances where non-native speakers associate a different pragmatic force to a particular utterance be that a speech act or a meaning of statement; or when a non-native speaker apply his native language norms and practices to the target language he/she is destined to misconstrue and be misconstrued by others. As a consequence, the pragmatic failure is invoked upon transferring syntactic or semantic equivalents with a different pragmatic force to the target language. The latter occurs due to the failure of non-native speaker being able to recognize when, why, and to whom along with the social context in which the utterance is produced.

Thomas (1983) puts great emphasis on knowing the social context of any speech act for non-native speaker to understand. This involves that non-native speakers have to develop considerable competence in the pragmatic aspects of the target language in order for them to construe utterances or speech acts intelligibly and coherently. Thomas (1983) talks about two types of competences: linguistic competence which deals with core linguistic matters. He claims that this type of competence is context-free; it is not concerned with the context in which utterances or speech acts occur. The other type of competence is non-linguistic or pragmatic one which allows non-native speakers to understand the intended meaning of any communicated utterances be that an idiomatic or formulaic expression or a speech act. Communication breakdown occurs when the intended meaning of the nonnative speaker's message is misconstrued by native speaker according to Beebe \& Takahashi (1989). The difficulty which ESL/EFL students encounter with idioms is that they pay a lot of attention to the linguistic component at the expense of the pragmatic one and by virtue of that they are destined to misconstrue the intended meanings of the idiomatic expression.

The findings of previous studies on the performance of speech acts cross-culturally have emphasized the following two important conclusions which are worthy of citation: a) that proficient learners are very likely to commit negative pragmatic transfer by using the norms and thoughts of their first language in the target language on account that they have developed a high proficiency level of the target language ( Kasper and Rose, 1996; Takashi and Beebe, 1987, 1993; El-Samaty, 2005; EslamRasekh,2004) B) That EFL learners with low proficiency in the target language are very likely to commit more negative pragmatic transfer than EFL learners with high proficiency due to their lack of proficiency in the target language (Takashi \& Dufon,1989; Robinson, 1992; Takahashi, 1996); Consequently, the findings of studies on the application of speech act cross-culturally point out that inter-language transfer occurs when EFL students fail to adhere to either or to both of the above instances.

Since the notion of culture appears to have great impact on one's perception, then an adequate amount of time has to be allocated to the question of culture for EFL students to consider seriously particularly when it comes to the acquisition/translation of idiomatic/formulaic expressions. This involves the diversification of ESL/EFL curriculum along with the pedagogy being used to afford 
students the opportunity to tap on their resources to maximize their overall understanding of the type of competence needed to use such expressions as coherently as possible.

There is a great deal of wisdom which one can draw from Edward Hall's (1976) notion of "high and low context" which underlies the impact of culture on inter or cross-cultural communication between/among people of different cultural and linguistic backgrounds. Lack of cultural knowledge of the target language can potentially constitute a real impediment to having a constructive communication. The findings of intercultural and cross-cultural communication studies stand as a genuine testimony to the impact of culture on ESL/EFL students' perception of various forms of linguistic structures and idiomatic expressions.

\subsection{Inter-Lingual Transfer}

Drawing on perspectives directly pertaining to both native speakers' and EFL/ESL students' errors and mistakes like Contrastive Analysis, Error Analysis, and Inter-lingual Analysis (referred hereto as CA, ER, and IL) becomes necessary to account fully for the type of errors manifested in EFL/ESL students' encounters with idiomatic/formulaic expressions. These three approaches or perspectives whose primary focus is EFL/ESL students' linguistic performance are usually looked upon as approaches whose primary concern is to account and explain the nature of the TL learner's performance and provide explanatory hypotheses concerning the type of errors committed by EFL/ESL learners and their source.

For instance Richards (1974) provides an insightful dichotomy between "errors" and "deviations" when one deals with EFL/ESL students' linguistic performance. According to him deviations can be accounted for in their context while errors are committed as a result of breaking a linguistic code of the same language. He claims further that deviations are "reflections of inter-lingual creativity". Obviously, the type of errors committed by ESL/EFL students in this study is definitely not linguistic errors and therefore they belong to a different category of errors/mistakes that have to be studied thoroughly and taken care of since their nature is totally distinct.

Other linguists like Brown (2000) and James (1998) have provided an interesting distinction between mistakes and errors. For example, producing imperfect sentences such as "Gorge speak English well instead of " Gorge speaks English well" as a result of tiredness on the part of first/second language learner is so frequent and is an indication of committing a mistake. The second type of deviation according to Brown (20000) and James (1998) results from a breaking of a specific linguistic code of the first or second language learner's linguistic system. Such dichotomy is very insightful concerning the process of first or second Language acquisition since it accounts not only for the deviation but also of its type and by virtue of that the situation can be accounted for and corrected.

IL is a term coined and adopted by Selinker (1972) which refers and describes the second language learner's linguistic system. IL has become an insightful perspective in language acquisition theory since it is concerned with the countless errors and mistakes which first and second language learners make which can't be attributed to differences or interference of one's first language or the acquisition of second language. This perspective focuses on the type of linguistic structures which the learner produces in his acquisition of first or second language but resembles neither his native language nor his target one. This particular perspective is highly insightful and revealing since it focuses on pointing out certain linguistic phenomena which are very likely to manifest themselves by the second language learner upon acquiring the target language which other perspectives on second language can't account for. Based on students' responses in this study it appears that the errors and mistakes committed by some of them can be attributed to their being unable to draw out the distinction between conventional and non-conventional meaning. The insightful thing in this context is that only the nonconventional meaning applies to idiomatic expressions.

\section{THE SignifiCANCE OF THE STUdY}

Time and again, formulaic/idiomatic expressions are an important topic that needs to be emphasized on account that there has been a scarcity of published data-based studies which focus on formulaic sequences in L2. Another important observation that has to be drawn from this study is that the subjects/informants of this study share so many common features in terms of their age, their initial exposure to and their comparable linguistic proficiency in the target language. As a consequence the findings of this study point out a representative pattern which speaks unequivocally of some conformity of undergraduates' responses. 
The significance of this study stems from the fact that it underlines the possibility that idiomatic expressions can be collapsed in the so called 'marked category' rather than the 'unmarked category' of any language on account that they are recurrent and have limited distribution and require specific type of knowledge to be processed; thus they are different from other unmarked linguistic forms/structures and consequently they constitute a real challenge to both native and non-ESL/EFL students.

This study has also underscored the reasons behind the adoption of a specific translation strategy over others and has provided the rationale behind ESL/EFL students' opting for the literal translation strategy over other strategies which ESL/EFL students are inclined to use to account for the translation of idiomatic/formulaic expressions. Furthermore, the findings have also underscored the instances which induce ESL/EFL students to resort to their native language as a communication strategy to account for the same task.

\begin{tabular}{|l|l|l|l|l|l|}
\hline Idioms & $\begin{array}{l}\text { Partial/full. Lexical } \\
\mathbf{8} \text { semantic } \\
\text { correspondence \% }\end{array}$ & $\begin{array}{l}\text { Literal } \\
\text { translation } \\
\text { with sense \% }\end{array}$ & $\begin{array}{l}\text { Literal } \\
\text { translation } \\
\text { with no sense } \\
\text { \% }\end{array}$ & $\begin{array}{l}\text { Paraphr } \\
\text { asing \%ot }\end{array}$ & $\begin{array}{l}\text { Not } \\
\text { answered } \\
\%\end{array}$ \\
\hline 1- in the twinkle of an aye & 63 & & 5 & & 3 \\
\hline 2- A stone's throw & 4 & & 67 & & a \\
\hline 3- Give him a free hand & 37 & & 33 & & 1 \\
\hline 4- Act of god & 37 & & 33 & & \\
\hline 5- Afraid of his own shadow & 67 & & 3 & & \\
\hline 6- Leave on stone unturned & 15 & & 55 & & 1 \\
\hline 7- Go whistle on the sea & 47 & 6 & 17 & & 4 \\
\hline 8- warm the heart & 8 & 32 & 30 & & 3 \\
\hline 9- Add fuel to the fire & 40 & 15 & 12 & & 1 \\
\hline 10- Under the banner of & 38 & 1 & 32 & & \\
\hline 11- Dig his own grave & 61 & 4 & 3 & & 19 \\
\hline 12- Up to his eyes & 8 & 24 & 38 & & \\
\hline 13- Walk on air & 10 & 25 & 35 & & \\
\hline 14- Carried the torch & 18 & 7 & 27 & & \\
\hline 15- - $0 v e$ to bag a trophy & & 26 & 45 & & \\
\hline $\begin{array}{l}\text { 16- USA is biting more than it } \\
\text { can chew }\end{array}$ & & 40 & 31 & & \\
\hline 17- Stick and carrot policy & & 63 & 8 & & \\
\hline 18- Golden age & & 52 & 15 & 2 & \\
\hline 19- Hidden agenda & & 34 & 36 & & \\
\hline 20- Take a back seat & & 60 & 11 & & \\
\hline
\end{tabular}

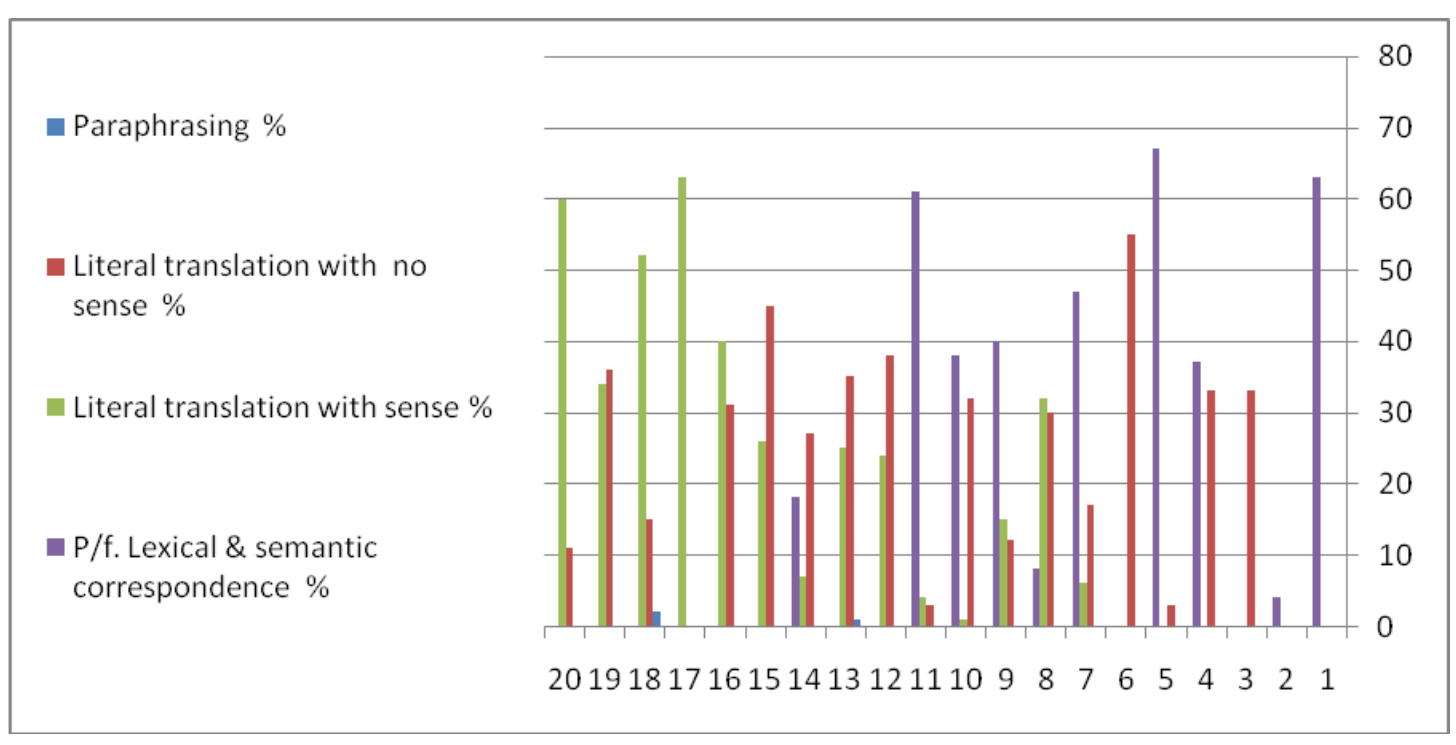

\section{Methodology}

The test being used in this study consists of 20 idiomatic expressions from the Oxford Dictionary of Idioms (2004) and cited in Abu-Ssaydeh's study (2004). It is designed to point out whether there are significant differences in the translation of idiomatic expressions which have and don't have 
equivalents. This test is administered to undergraduate English major at An-Najah National University. It is designed to point out whether there are significant differences in the translation of idiomatic expressions which have equivalents and those which do not. The second component was a follow-up conducted by the researcher to follow up on the students' responses and for the sake of soliciting necessary feedback on the causes behind the difficulties students encounter with the translation of such expressions.

\section{RESUlTS AND DisCUSSION}

According to Jaszczolt (1995) Cross Linguistic Interference known as CLI is an important factor to consider in language acquisition. The proponents of such hypothesis claim that the influence can be on both from and to native and target language. The findings of this study have pointed that idiomatic expressions which have equivalents in the informants' first language would be easier to handle than the ones which have no equivalents and by virtue of that the latter group poses a real challenge to the majority of informants. So the idiom, "He twisted his arm" is easy to do because students can appeal to its literal(semantic) meaning or find its full equivalent in the target (native)language whereas the idiom "To bring home the bacon and Like a red rag to a bull", have no equivalents and therefore they constitute a real problem.

Such influence has manifested itself in the students' translation of most idiomatic expressions. Therefore, the findings seem to support the assumption that prior experience has a major role to play in any act of learning or in the acquisition of knowledge which in turns inclines one to believe that one's native language should be taken into consideration. Keshavarz's (1994) taxonomy of the sources of errors has underscored the possibility of inter-lingual errors resulting from the transfer of linguistic elements of the learner's mother tongue to the learning of the target language.

The findings of this study confirm a set of conclusions which have to be taken into account when ESL/EFL students deal with idiomatic expressions. Initially, idioms belong to several different species and therefore they require different translation strategies to fully account for their precise translation. A second observation that appears to be operative among students is that they opt for the literal translation unless they know the proper translation strategy for the idiom they are dealing with. A third observation which manifested itself in their responses is that when ESL/EFL students come across an idiom in the target language which has equivalent in the source language, they are very likely to attend to it successfully. For instance in set A, which includes idioms 1 through 5, where students are expected to supply their full equivalence in their native language, we noted that the majority of them have attended successfully to this set of idioms (i.e. from 1-5) with some exception to idiom number 2 on account that their lack of exposure to such an idiom has probably prevented students to recognize that such an idiom has full correspondence in their native language. And by virtue of that idiom number 2 has constituted a real challenge to the majority of students.

In set $\mathrm{B}$, which includes the idioms 6-13, the situation is different since the idioms in this particular set require a semantically or lexically partial correspondence in the target language. The findings have supported the claim that when there is partial correspondence between the source and target language with regard to a specific idiom, ESL/EFL students find it relatively possible to attend to such idioms with some difficulty. However, the findings point out that due to lack of use and exposure, ESLEFL students continue to experience a lot of difficulty in tackling such idioms. Great deal of discrepancy has manifested in their responses. For instance students were able to attend to some of the idioms but not as adequately as the ones in set A. For instance, we noted that the majority of students appealed to the literal meaning to account for the translation of idioms in set B (idioms number 6-10 ), even though they have partial correspondence in their native language. Such a result is likely to incline one to infer that when ESL/EFL students encounter an idiomatic expression they resort to their native language as a potential strategy to account for its translation. Another tenable explanation which one can infer is that due to lack of use and little exposure to such idioms in English, ESL/EFL students are prone to opt for the literal translation as a potential option. Such findings have been consistent with those of Abu-Ssaydeh's (2004) study despite the fact that his study has dealt with professional translators whereas the informants of this particular study are college students whose linguistic proficiency appears to be lacking due to various reasons one of which is their lack of exposure to such idioms and their being deprived from the opportunity to use such idioms in real situations. 
Another striking observation which draws one's attention is that they didn't fare well with idioms number 6 and 8 since they provided the literal translation of these two idioms. That is to say, they failed to take into account that these two idioms call for partial correspondence in the target language. Instead of paying attention to the semantic correspondence, they literally translate each lexical item. This is an important matter that English major seem to fall into when they confuse the conventional with the non-conventional meaning of idioms. Such a challenge seems to persist throughout their account of the idioms in set B, and appears to be pervasive in their responses to such idioms.

When students attempted to translate idiom number 13, they supplied the literal meaning of the words which constitute it and by virtue of that they have misconstrued the intended meaning or point of this particular idiom due to lack of exposure and use. They just supplied the literal meaning of the lexical words of this idiom and that is an indication of confusing the conventional with the non-conventional meaning if such an idiom.

In set $\mathrm{C}$, which includes idioms from 14-20, the situation differs. Students were expected to pay a great deal of attention to the literal meaning of such idioms so long as their rendition of such idioms is consistent and makes senses. When attempted to translate idioms in set $\mathrm{C}$, they supplied the literal sense of all the idioms in which some of their responses made sense but others did not. They provided the literal meaning of most of the remaining idioms. In set $\mathrm{C}$, the findings seem also to confirm the assumption that appealing to the literal meaning of the idioms in set $\mathrm{C}$ was the easiest task but the apparent challenge for students in tackling such idioms is again their lack and use of these idioms have constituted the primary problem. For example idioms 14 and 15 seem the most challenging ones in set $\mathrm{C}$ on account that they are totally unfamiliar and require some sense.

Idioms $17,18,19$, and 20, appear to be familiar and students have attended to them with ease and care. We can attribute the successful attempts made by the majority of students in tackling the last four idioms to the fact that there is some correspondence between the semantic and literal meaning of these idioms. In 14 and 16 students appealed to their literal meaning and as a result their rendition sounded senseless. Again, a persistent pattern that appears to run through their responses is their reliance on literal translation as a tenable translation strategy to account for anything. This inclines one to claim that due to lack of use and exposure to some of these idioms students continue to encounter a great deal of difficulty when they try to account for the translation of such idioms. Finally and most importantly is that finding the proper and suitable translation strategy is a real challenge to the majority of college English major.

Based on the information which I have solicited from my informants (students) after taking the test, the real challenge that students seem to face when dealing with idioms is not only finding out the proper translation strategy for a specific idiom but also being able to deal with different idioms which require the use of different translation strategies. A quick glance at students' responses is likely to convince one that what appears to have compounded the problem further is the tendency of college students to opt for "literal translation strategy" as the safest resort to fall back on in the face of so many diverse types of idioms whose conventional meaning differs from non-conventional one, a real challenge which is bound to continue.

\section{CONClusion}

Pedagogically speaking, it is very clear that acquiring a foreign language requires not only the acquisition of a linguistic system but rather a continuous exposure to the target culture since most of the problems encountered by ESL / EFL students to account for the translation of idiomatic/formulaic expressions are culture bound and require the acquisition of pragmatic competence (see, Frank 2013). The findings of cross-cultural studies on language acquisition and speech act theory underscore the impact of culture on ESL students' perception of the target language. The findings of this study have pointed out that when EFL/ESL students translate an idiomatic/formulaic expression which has no equivalence in their mother tongue, they encounter a great deal of difficulty and are very likely to opt for its semantic equivalence or literal translation.

This proves that EFL/ESL students resort to their mother tongue for assistance to accommodate their immediate needs which in turn proves the validity and merits of the CLI and CAH in second language acquisition. The findings of this study have also underscored the merits of the MDT as an instrumental mechanism in assisting ESL/EFL students and instructors in distinguishing the differences between the 'marked versus 'unmarked' forms/structures of any linguistic system. 
Furthermore, and most importantly, the findings of this preliminary study have clearly shown that ESL/EFL students have approached the task of translating formulaic/idiomatic expressions as if such expressions were real linguistic forms/structures and by virtue of that most students resorted to interlingual transfer for assistance to account for the rendition of idiomatic expressions.

Moreover, exploring the merits of applying the MDT and UG as an effective pedagogical framework to account for other types of marked structures or in any particular language is of great value to the applied and theoretical linguists. And finally and more importantly is that idioms are linguistically and cultural complex and specific and so are the selection of the optimal translation strategy ESL/EFL students. In fact we are still curious to know whether the acquisition of such expressions requires both native and non-native speakers to tap on different mental resources like the right hemisphere of one's brain as Obler's study indicated (1982) or not since they are too distinct from any typical linguistic utterance. Only by carrying out much more intensive and extensive research on the acquisition of 'marked forms' or ' non-canonical' forms, we would be able to explore the validity of such proposition and that of Obler's

\section{REFERENCES}

Abu-Ssaydeh, A. (2004) The translation of English idioms into Arabic. Babel, 50 (20, 114-131.

Al-Khateeb, Sana. (2009) " The speech act of thanking as a compliment response as used by the Arab speakers of English -a comparative intercultural study" Unpublished M.A. thesis, An-Najah National University, Nablus, West Bank, Palestine.

Awwad, M.(1991). "Equivalence and the translatability of English and Arabic idioms" PSiCL. Vol.26,: 57-67.

Baker, M. (1992). In other words: A Course on translation. London: Rutledge

Beebe L. Takashi T.: (1989) "Sociolinguistic variation in face-threatening acts" in Eisenstein M. R. ed. The Dynamic Inter-lingual Empirical Studies in Speech Variation. New York: Plenum Press.

Brown, H. Douglas. (2007). Teaching principle: An Interactive Approach to language pedagogy. Pearson Education ESL: 3 edition

Chomsky, Noam. (1986). Knowledge of language. Its nature, origin, and use. Praeger: New York.

Chomsky, Noam. (1993) language and thought. Group West.USA

Comrie, Bernard. (1990). "Second language acquisition and language universals research" Studies in Second Language Acquisition 12: 209-218.

Ekman, Fred R. (1977). "Markedness and the contrastive analysis hypothesis" Language Learning 27: 315-330.

El-Samaty, M.: (2005), "Helping foreign language learners become pragmatically competent", proceedings of the $10^{\text {th }}$ TESOL Arabia Conference,9.

Eslami-Rasekh Z., Eslami-Rasekh A. and Fatahi A.: (2004), The effect of explicit metapragmatic instruction on the speech act awareness of advanced EFL students". TESL-EJ (8), 2. Retrieved from http://www-writing .berkeley.edu/TESI-EJ/ej30/a2.html.

Fillmore, C.J. (1979). In C.J. Fillmore, D. Kempler \& S.-Y.W. Wang (eds.) Individual differences in language ability \& language behavior. New York: Academic Press, 85-101.

Frank, Jerrold. (2013). "Raising cultural awareness in English language classroom" English teaching forum. Vol 51, N 4. 2-11.

Hall, Edward. (1976). Beyond Culture. New York: Anchor Press.

Hymes, D.H. (1968). The ethnography of speaking. In T. Gladwin \& W.C. Sturtevant (eds.) Anthropology and Human Behavior. Washington, D.C.

Robinson, M. S.: (1992) "Introspective methodology in inter-language pragmatics research ", in Kasper G. ed. Pragmatics of Japanese as Native and Target Language. Second Language Teaching Curriculum Center: University of Hawaii at Monoa.

Gass, S. and Selinker, L. (1983) Language transfer. In F. Eppert (ed.) Transfer and translation in Language Learn. Singapore: SEAMEO

Jaszczolt, Katarzyna. (1995) "Typology of contrastive studies: Specialization, progress, and application" Language Teaching, 28: 1-15.

Kasper G. and Schmidt R.: (1996) "Developmental issues in inter-language pragmatics". Studies in Second Language Acquisition, 18, 149-169. 
Kharma, N. and Hajjaj, A. (1997) Errors among Arab speakers: Analysis and remedy. Beirut: Libraire du Liban.

Lado, Robert. (1957). Linguistics across cultures. Ann Arbor: University of Michigan Press.

Lewis, M. (1998). Implementing the lexical approach. LTP. England. Pp. 223.

Longman Idioms Dictionary. (1998). UK: Longman, English. Pp. 398.

Mahmoud, A. (2002) The Inter-lingual errors of Arab students: A course book education English majors. Unpublished manuscript, Sultan Qaboos University, Oman

Obler, Lorraine K. 1982. Neurolinguistic aspects of language loss as they pertain to second language acquisition. In Lambert \& Freed 1982.

Odlin, T. (1989). Language transfer. Cambridge, NY: Cambridge University Press.

Pawley, A. \& Syder, F.H. 1983. Two puzzles for linguistic theory: native-like selection and nativelike fluency. In J.C. Richards \& R.W. Schmidt (eds.)

language and communication. New York: Longman, 191-226.

Richards, Jack C. (1974). Error analysis: Perspective on second language acquisition. London: Longman Group, Ltd.

Awwad, M. (1991). "Equivalence and translation of English and Arabic idioms" PSiCL. Vol. 26,: 57-67.

Pinker, S. 1994. The language instinct. Harmondworth: Penguin.

Sequeiros, R. Xose..(2004). Formulaic expressions in translation. Trans. No 8

Sperber, D. \& Wilson, D. (1995). Post face to the second edition of Relevance:

Communication and Cognition. Oxford: Blackwell.

Selinker, Larry. 1972. " Inter-language" International Review of Applied Linguistics. 10: 201-231.

Takahashi, S.: (1996), "Pragmatic transferability" Studies in Second Language Acquisition, 18, 189-223.

Takahashi T. and Beebe L, M.; (1987) "The development of pragmatic competence in Japanese learners of English", JALT Journal, (8).

Takahashi T. and Beebe L. M. ; (1993), "Cross-linguistic influence in the speech act of correction", in Kasper G. and Blum-Kulka S. (eds). Inter-language Pragmatics. New York: Oxford University Press.

Takahashi, S. and Dufon, M.A.: (1989) "Cross-linguistic influence in indirectness: the case of English directives performed by native Japanese speakers" unpublished paper, University of Hawaii at Monoa.

Thomas, J. (1983). Cross-cultural pragmatic failure. Applied Linguistics Vol. 4 (2),:91-112.

Wray, Alison. (2002). Formulaic language and the lexicon. Cambridge: Cambridge University press.

\section{AUTHOR's BIOGRAPHY}

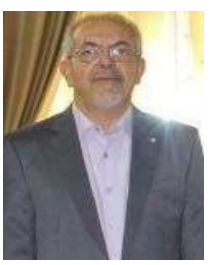

I am an assistant professor of linguistics and speech communication at An-Najah National University on the West Bank in Palestine. I have been teaching there for more than twelve years. Over the past ten years, I have held many administrative positions in addition to my being an academic. As an academic, I have held many administrative positions at the university. I have worked as chair of the English Department and chair of the Graduate Programs in Humanistic Studies at the college of Graduate Studies at An-Najah University.

As an instructor, I have had a wide range of teaching experience whether at home or abroad in the U.S.A. I have been teaching undergraduate and graduate courses in general linguistics, discourse analysis, sociolinguistics, and speech communication. I have also been instrumental in supervising more than a dozen M.A. theses in our M.A. Program in Applied Linguistics and Translation at anNajah.

By and large, I am inclined to describe myself as a bi-cultural linguist. I have obtained an M.A. in Applied linguistics from the State University of New York at Stony Brook and an interdisciplinary Ph.D. degree in humanistic studies, intercultural communication, and general linguistics at the University of Albany. 